\title{
A SECOND CASE OF OUTBURSTS IN A PULSATING WHITE DWARF OBSERVED BY KEPLER
}

\author{
J. J. Hermes ${ }^{1}$, M. H. Montgomery ${ }^{2}$, Keaton J. Bell ${ }^{2}$, P. Chote ${ }^{1}$, B. T. Gänsicke ${ }^{1}$, Steven D. Kawaler ${ }^{3}$, \\ J. C. Clemens ${ }^{4}$, Bart H. Dunlap ${ }^{4}$, D. E. Winget ${ }^{2}$, and D. J. Armstrong ${ }^{1}$ \\ ${ }^{1}$ Department of Physics, University of Warwick, Coventry CV4 7AL, UK; j.j.hermes@warwick.ac.uk \\ ${ }^{2}$ Department of Astronomy, University of Texas at Austin, Austin, TX 78712, USA \\ ${ }^{3}$ Department of Physics and Astronomy, Iowa State University, Ames, IA 50011, USA \\ ${ }^{4}$ Department of Physics and Astronomy, University of North Carolina, Chapel Hill, NC 27599-3255, USA \\ Received 2015 June 14; accepted 2015 July 21; published 2015 August 24
}

\begin{abstract}
We present observations of a new phenomenon in pulsating white dwarf stars: large-amplitude outbursts at timescales much longer than the pulsation periods. The cool $\left(T_{\text {eff }}=11,060 \mathrm{~K}\right)$, hydrogen-atmosphere pulsating white dwarf PG 1149+057 was observed nearly continuously for more than 78.8 day by the extended Kepler mission in $K 2$ Campaign 1 . The target showed 10 outburst events, recurring roughly every 8 day and lasting roughly $15 \mathrm{hr}$, with maximum flux excursions up to $45 \%$ in the Kepler bandpass. We demonstrate that the outbursts affect the pulsations and therefore must come from the white dwarf. Additionally, we argue that these events are not magnetic reconnection flares, and are most likely connected to the stellar pulsations and the relatively deep surface convection zone. PG $1149+057$ is now the second cool pulsating white dwarf to show this outburst phenomenon, after the first variable white dwarf observed in the Kepler mission, KIC 4552982. Both stars have the same effective temperature, within the uncertainties, and are among the coolest known pulsating white dwarfs of typical mass. These outbursts provide fresh observational insight into the red edge of the DAV instability strip and the eventual cessation of pulsations in cool white dwarfs.
\end{abstract}

Key words: stars: evolution - stars: general - stars: individual (PG 1149+057) - stars: oscillations - stars: variables: general - white dwarfs

\section{INTRODUCTION}

The vast majority of all stars in our Galaxy will end up as white dwarfs, which provide important observational boundary conditions on the final stages of stellar evolution. White dwarfs have electron-degenerate cores overlaid by a thin, nondegenerate atmosphere, and passively release their residual thermal energy. When a DA (hydrogen-atmosphere) white dwarf eventually cools below $12,500 \mathrm{~K}$, it develops a hydrogen partial-ionization zone at its surface. This superficial convection zone throttles heat transport and efficiently drives pulsations (Brickhill 1991a; Goldreich \& Wu 1999a).

The flux changes in variable DA white dwarfs (DAVs, aka ZZ Cetis) are consistent with surface temperature variations caused by non-radial $g$-mode pulsations. By matching the observed pulsation periods to theoretical models, we can reveal considerable detail about the interior of white dwarfs, including their overall mass, temperature, the mass of their outer hydrogen and helium envelopes, their rotation, and even characteristics of their cores (see reviews by Fontaine \& Brassard 2008; Winget \& Kepler 2008; Althaus et al. 2010).

As white dwarfs cool their convection zones deepen. Since the oscillation modes excited are characterized by the thermal timescale of the driving zone, a deeper convection zone excites long-period pulsations, up to an observed maximum of about $1200 \mathrm{~s}$ for a $0.6 M_{\odot}$ white dwarf (Mukadam et al. 2006). Empirical studies of the coolest members of the DAV instability strip are complicated by the relatively long-period and less coherent pulsations (Kanaan et al. 2002). However, the Kepler space telescope provides an unprecedented tool for long-term monitoring of the coolest pulsating white dwarfs.

Ten pulsating white dwarfs were eventually discovered late in the original Kepler mission, but only two were observed for more than a month before the failure of the second reaction wheel in 2013 May (Hermes et al. 2011; Greiss et al. 2014). Curiously, the first and longest-studied DAV observed by Kepler, KIC 4552982, showed a completely unprecedented behavior: large-amplitude flux excursions which raised the overall brightness by up to $17 \%$, recurred on average every 2.7 days and lasted $4-25 \mathrm{hr}$ in duration (Bell et al. 2015). These outbursts recurred on a much longer timescale than the pulsations.

The extended Kepler mission (K2) affords an exceptional opportunity to extend space-based monitoring for up to 85 days to a plethora of new (and brighter) white dwarfs (Howell et al. 2014). Already $K 2$ has illuminated frequency variability in the cool DAV GD 1212 (Hermes et al. 2014) and a hot DAV descended from common-envelope evolution (Hermes et al. 2015).

The pulsating white dwarf PG $1149+057$ was observed for more than 78.8 days in $K 2$ Campaign 1 , and we present here an analysis of the second case of sporadic, high-amplitude outbursts in a cool DAV. In this letter we present our $K 2$ observations, establish that the outbursts are happening on the white dwarf, and analyze the outbursts and their relation to the stellar pulsations.

\section{K2 CAMPAIGN 1 TIME-SERIES PHOTOMETRY}

There is little high-speed photometry on PG $1149+057$, despite the fact that there are just 15 brighter known DAVs. Pulsations were announced using one very short $(<0.7 \mathrm{hr})$ run in 2005 May by Voss et al. (2006), who present the only previously published light curve.

Our K2 Campaign 1 coverage of PG 1149+057 (EPIC $201806008, K_{p}=15.0 \mathrm{mag}$ ) was requested in short-cadence (exposures every $58.8 \mathrm{~s}$ ) and spans from 2014 June 3 00:00:42 UT to 2014 August 20 20:16:36 UT. Every 6 hr, the Kepler 

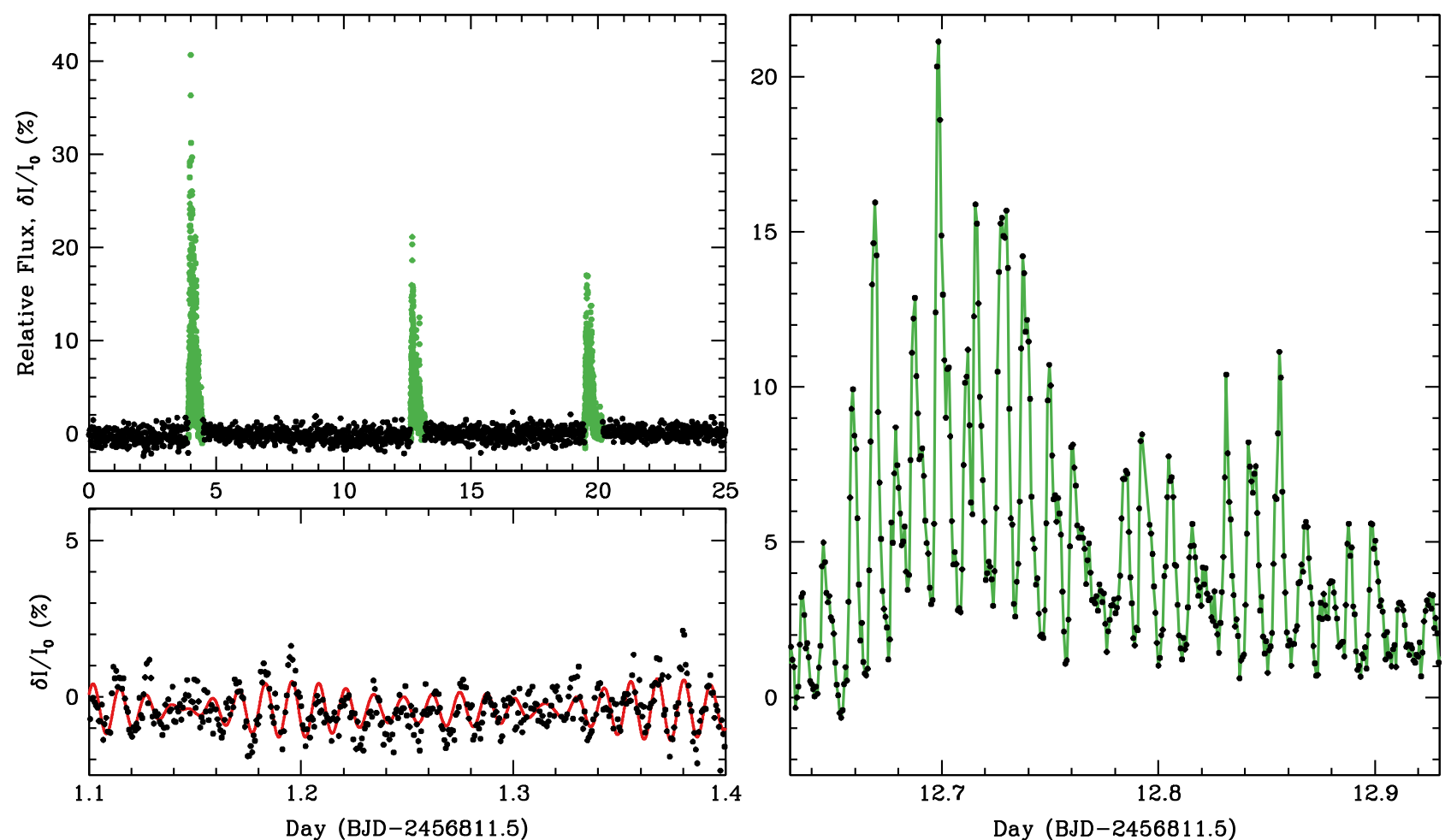

Figure 1. Representative portions of the $K 2$ Campaign 1 light curve of the pulsating white dwarf PG $1149+057$. The top left panel shows the first 25 days of observations; three outburst events are denoted in green. The bottom left panel shows $7.2 \mathrm{hr}$ of data on the second day of $K 2$ observations; the white dwarf pulsations are clearly visible, and underplotted is a best-fit to the three highest-amplitude signals (with periods of 1145.7, 998.1, and 1052.8 s). The right panel shows $7.2 \mathrm{hr}$ during the second outburst, with points connected in green.
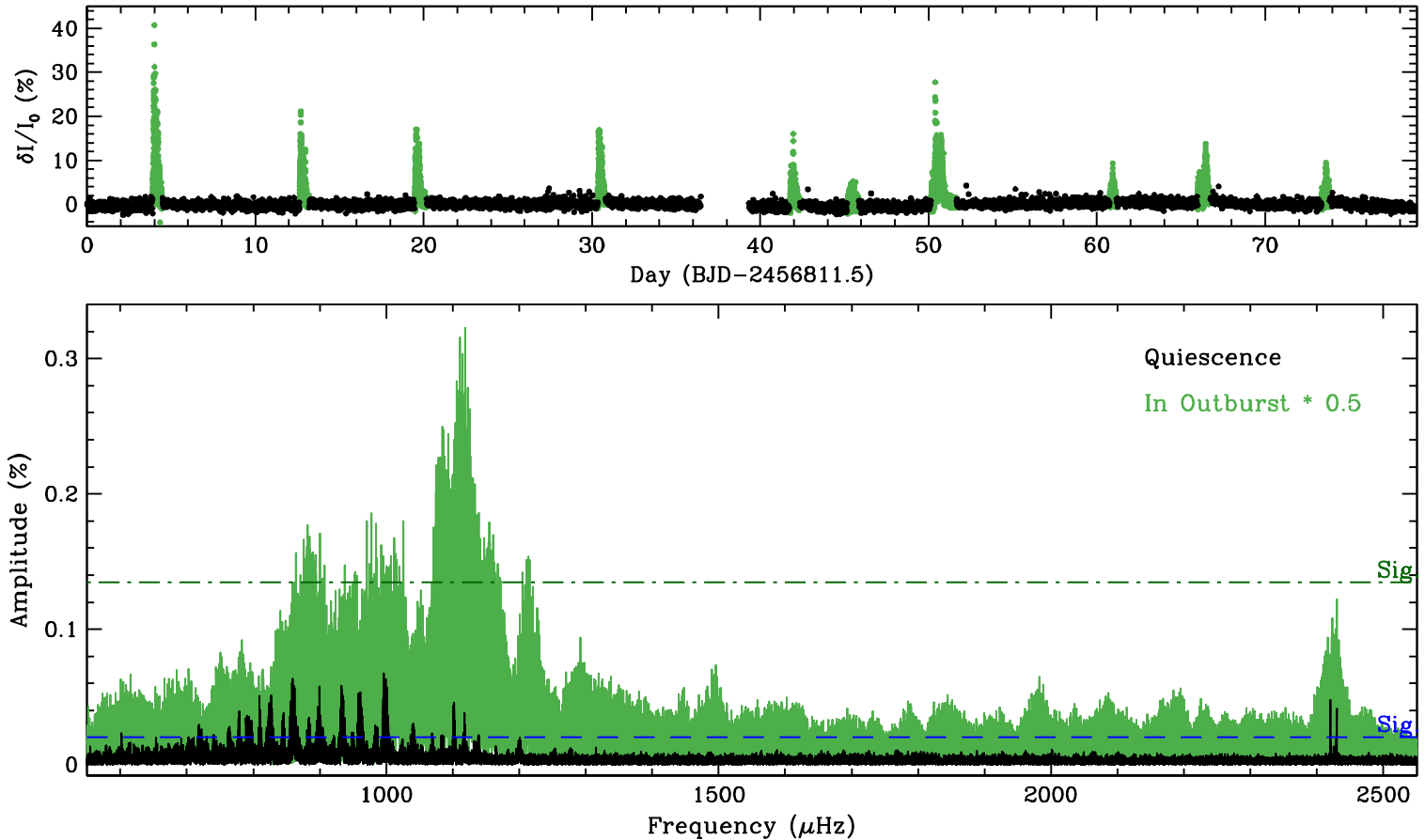

Figure 2. Top panel shows the entire $K 2$ light curve of PG $1149+057$. We mark the 10 outburst events in green and show the data in quiescence as black points. The bottom panel shows FTs both in (green) and out of outburst (black). Both subsets have pulsations excited above the significance threshold, described in the text and shown as dark green dashed-dotted and blue dashed lines, respectively. Pulsations persist during outburst but have higher amplitudes and shorter periods than in quiescence.

spacecraft checks its pointing and, if it needs correction, fires its thrusters. This can cause significant discontinuities in the light curve. We have extracted and detrended the photometry using the tools outlined in Armstrong et al. (2015), which compensates for artifacts in the light curve caused by attitude corrections. 
Our final data set has 107,443 points, yielding a duty cycle $>92.8 \%$. Figure 1 shows the first 25 days of $K 2$ coverage, including the first three of a total of 10 outbursts. Inspecting the light curve in more detail reveals the 1\%-3\% peak-to-peak variability caused by the white dwarf pulsations.

We display the entire $K 2$ light curve in the top panel of Figure 2, marking each outburst in green. The multi-day gap beginning near 2456849 BJD was caused by a major pointing shift in the spacecraft, which subsequently added minor, longtimescale variations about the mean flux level.

We have inspected movies of the Kepler images around each of these outbursts using the K2FLIX package (Barentsen 2015) and see no evidence for passing solar system bodies causing brightness increases. Kepler has a large plate scale (4 arcsec pixel $^{-1}$ ), but the nearest source with $r<19.5 \mathrm{mag}$ is SDSS J115155.09+052959.3, which is more than 1.3 arcmin to the north, well outside the extracted target pixels.

\section{ARCHIVAL SPECTROSCOPY AND PHOTOMETRY}

We describe here the time-averaged atmospheric parameters for PG $1149+057$, and exclude the possibility that this white dwarf has a flaring, low-mass companion.

To date, the most consistent picture of the parameters of white dwarfs in and around the DAV instability strip has been undertaken by Gianninas et al. (2011). Their one-dimensional, ML2 $/ \alpha=0.8$ atmospheric parameters place PG 1149 +057 near the empirical red edge of the DAV instability strip, with $T_{\text {eff }}=11,360 \pm 170 \mathrm{~K}$ and $\log g=8.21 \pm 0.05$. Tremblay et al. (2013) introduced terms to correct for the threedimensional (3D) dependence of convection, which refine the final determinations for PG $1149+057$ to $T_{\text {eff }}=11,060 \pm$ $170 \mathrm{~K}$ and $\log g=8.06 \pm 0.05$. This corresponds to a mass of $0.64 \pm 0.03 M_{\odot}$ and a distance of $39.3 \pm 2.4 \mathrm{pc}$ using the mass-radius relationship of Renedo et al. (2010).

High-resolution spectra taken as part of the ESO SNe Ia Progenitor Survey show no evidence of metals from ongoing accretion (Voss et al. 2006), and the radial velocities of this white dwarf do not vary to a limit of $4.0 \mathrm{~km} \mathrm{~s}^{-1}$ over $4 \mathrm{hr}$ (Maxted \& Marsh 1999). We have inspected the Wide Angle Search for Planets survey light curve of PG 1149+057, which covers more than 3 years but is at the faint end for the survey. Despite binning the data into 30 minute intervals, the $1 \sigma$ standard deviation of all points is $0.17 \mathrm{mag}$, so we cannot significantly constrain whether outbursts have been observed from previous ground-based studies.

We have examined the wealth of archival photometry on PG $1149+057$ and show in Figure 3 that the spectral energy distribution (SED) is consistent with a single, isolated white dwarf from the near-ultraviolet out to the mid-infrared. The SED uses photometry from GALEX-NUV (Morrissey et al. 2007), SDSS-ugriz and UKIDSS-JHK (Girven et al. 2011), and Spitzer 3.6 and $4.5 \mu \mathrm{m}$ photometry (Barber et al. 2012). Underplotted in Figure 3 is an $11,000 \mathrm{~K}, \log g$ $=8.0 \mathrm{DA}$ white dwarf model atmosphere computed by Koester (2010), normalized to the SDSS- $r$ band.

The lack of infrared excess as compared to our white dwarf model rules out any possible companion earlier than L4 using the data of Cushing et al. (2005). Our K2 light curve is therefore composed entirely of PG $1149+057$, and the outbursts observed are the result of brightening events on the surface of the white dwarf.

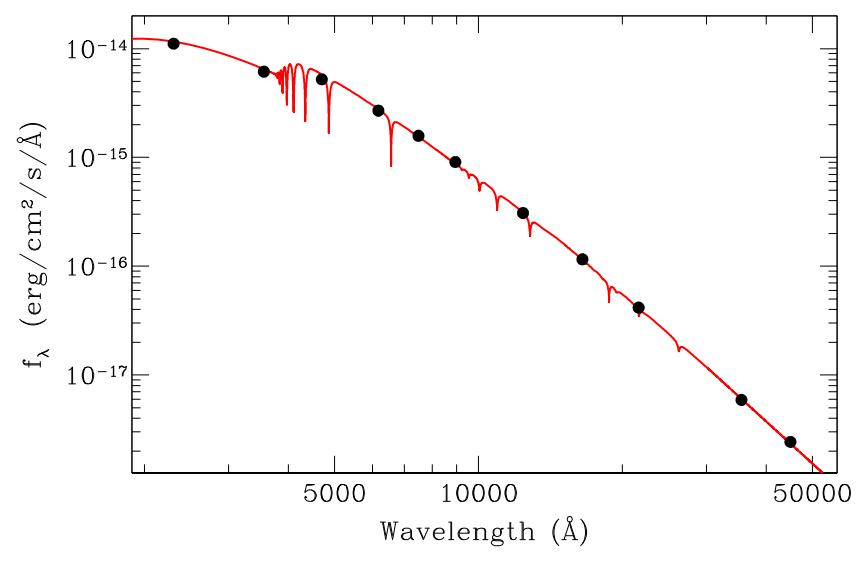

Figure 3. Spectral energy distribution from archival data demonstrates that there is no companion to PG $1149+057$ responsible for the outbursts. The data are consistent with an isolated white dwarf (this an $11,000 \mathrm{~K}$ model) from the near-ultraviolet out to the mid-infrared (see the text for sources). The $3 \sigma$ photometric uncertainties are smaller than each point.

\section{DISCUSSION OF THE OUTBURSTS}

\subsection{Outburst Characteristics}

The outburst events shown in the top panel of Figure 2 confirm a new phenomenon observed in pulsating white dwarfs. Only one other pulsating white dwarf displays similar recurring behavior: The cool DAV KIC 4552982 (WD J1916 +3938 ), observed for nearly 2.5 years in the original Kepler mission (Bell et al. 2015).

That DAV has very similar 3D atmospheric parameters to PG $1149+057-T_{\text {eff }}=10,860 \pm 120 \mathrm{~K}$ and $\log g=8.16 \pm$ 0.06 - and it also has a very similar pulsation spectrum. The only other reminiscent event seen in a pulsating white dwarf occurred during monitoring of the cool variable DB (heliumatmosphere) white dwarf GD 358, which in 1996 underwent a dramatic change in excited frequencies accompanied by a rapid increase in fractional amplitude, its sforzando (Montgomery et al. 2010).

The outbursts in KIC 4552982 increase its mean flux by between $2 \%$ and $17 \%$, last $4-25 \mathrm{hr}$, and recur on an average every 2.7 days (Bell et al. 2015). Everything about the outbursts in PG $1149+057$ is scaled up by roughly a factor of three. The outbursts here increase the quiescent flux by between $5 \%-45 \%$, have durations on average of $14.5 \mathrm{hr}$ (ranging from 9.3-36.4 hr), and recur on a timescale between 3.5 and 11.5 days, on average every 8 days. These rough timescales were determined by eye, noting when the point-topoint scatter rose and fell to the quiescent values. With so few events, an autocorrelation function reveals only that most events last more than $12 \mathrm{hr}$. There does not appear to be any periodicity to the outbursts. Each outburst requires an increase in energy from quiescence of roughly $10^{34} \mathrm{erg}$, found by integrating the mean equivalent duration of the outbursts of 24.8 minutes.

\subsection{Outbursts Affect the Pulsations}

We see convincing evidence that the pulsations persist on the white dwarf during the outburst, and actually change properties relative to those excited in quiescence. It is important to note that if these outbursts came from a contaminating source the pulsation amplitudes would decrease as a result of flux dilution. That the pulsation amplitudes actually increase proves that the outbursts are on the white dwarf. 

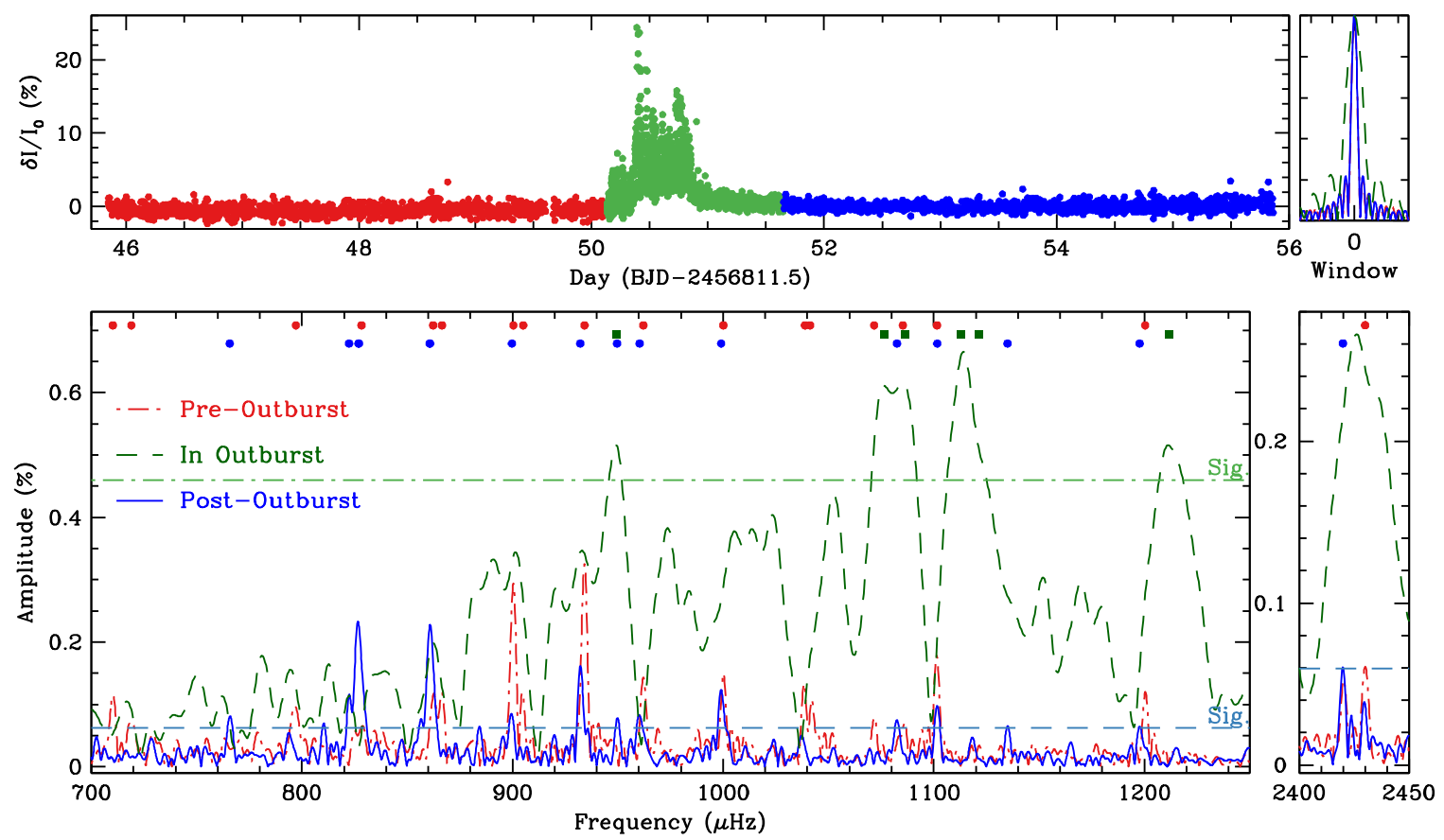

Figure 4. Diagnostics of the pulsations before (red), during (green), and after (blue) the seventh outburst observed on PG $1149+057$. The top panel shows the $K 2$ light curve, while the bottom panels show the FT of each subset. Signals exceeding the significance thresholds (see text) are marked with appropriate-colored dots and show differences in the excited pulsation spectra. Amplitudes are essentially all lower after outburst, but subsequently grow (see Figure 5). Significant pulsations are also visible during the brightening event (green squares), and appear at shorter periods (higher frequencies) and higher amplitudes. The top right panel shows the window function of each subset.

The bottom panel of Figure 2 shows Fourier transforms (FTs) of the entire light curve, differentiated by data in and out of outburst. Both datasets have peaks in the FT above the respective $3 \sigma$ significance threshold, which was determined by randomly shuffling the fluxes for each point in the light curve and computing the highest peak in the entire resultant FT for 10,000 random permutations (see Hermes et al. 2015).

Figure 2 shows that the pulsations in outburst have shorter periods and higher amplitudes than those in quiescence; the unweighted mean period before, during, and after the seventh outburst goes from $1083.3 \mathrm{~s}$ to $919.7 \mathrm{~s}$ to $1072.7 \mathrm{~s}$, respectively. If the flux increase during the outbursts are due to an increase in the average effective temperature of the star, the formalism of Wu \& Goldreich (1999) predicts that the surface amplitudes of the modes should increase, with the enhancement being greater for shorter-period modes. The $1000 \mathrm{~s}$ modes are expected to have growth times of order days (Goldreich \& Wu 1999a).

Smoothing the light curve by $3000 \mathrm{~s}$, over several pulsation cycles, the outbursts increase the mean flux by up to $14 \%$, which would require a global $750 \mathrm{~K}$ temperature increase from the quiescent effective temperature of $11,060 \mathrm{~K}$. The pulsations induce even more dramatic, localized temperature excursions.

Figure 4 explores the light curve surrounding and during the seventh observed outburst, between Days 46-56 from the start of $K 2$ monitoring, using an equal amount of data before and after the outburst. An FT of each subset clearly shows that pulsations are excited to significant amplitudes, even during the outburst.

Many of the significant signals in outburst are not present in quiescence, especially the mode at $949.3 \pm 0.8 \mu \mathrm{Hz}(1053.4 \pm$ $0.8 \mathrm{~s}$ ), which decreases in amplitude the longer we monitor it after outburst. Many modes appear to eventually return at roughly the same period after the outburst. However, the amplitude evolution of these pulsations holds key insight.

In essentially all cases in Figure 4, the amplitudes shown in the subset immediately after the outburst are lower than the preoutburst subset. There are more data in this post-outburst subset, and we can clearly see many modes growing after the outburst. We explore this amplitude evolution using a running FT, shown in Figure 5. We have computed this diagram by taking an FT across a sliding 3-day window, stepped every hour with $0.05 \mu \mathrm{Hz}$ frequency resolution.

This time-dependent FT demonstrates that the outburst has depleted considerable pulsation energy. The apparent resetting of amplitudes offers the unprecedented opportunity to actually measure the linear growth rates of pulsation modes in a white dwarf, although that is outside the scope of this Letter.

Finally, the bottom right panel of Figure 4 shows the relatively stable group of three symmetric signals centered at $2425.0 \mu \mathrm{Hz}$ (the $m= \pm 1$ components are visible in this short subset but not the central component). This pulsation triplet arises from rotation of the white dwarf (Pesnell 1985), from which we measure an $\ell=1$ frequency splitting of roughly $4.7 \mu \mathrm{Hz}$, which corresponds to a rotation period of roughly 1.2 days in the asymptotic limit. We reserve a full period determination and detailed asteroseismic analysis of the many pulsations in PG 1149+057 for a future publication.

\subsection{Consideration of Possible Outburst Mechanisms}

We consider various timescales in white dwarfs to explore different underlying mechanisms for the outbursts.

White dwarfs have very short dynamical timescales, roughly a few seconds. While this is a global estimate, we expect all processes which are not in hydrostatic equilibrium to locally occur on this or an even shorter timescale. The timescales for 


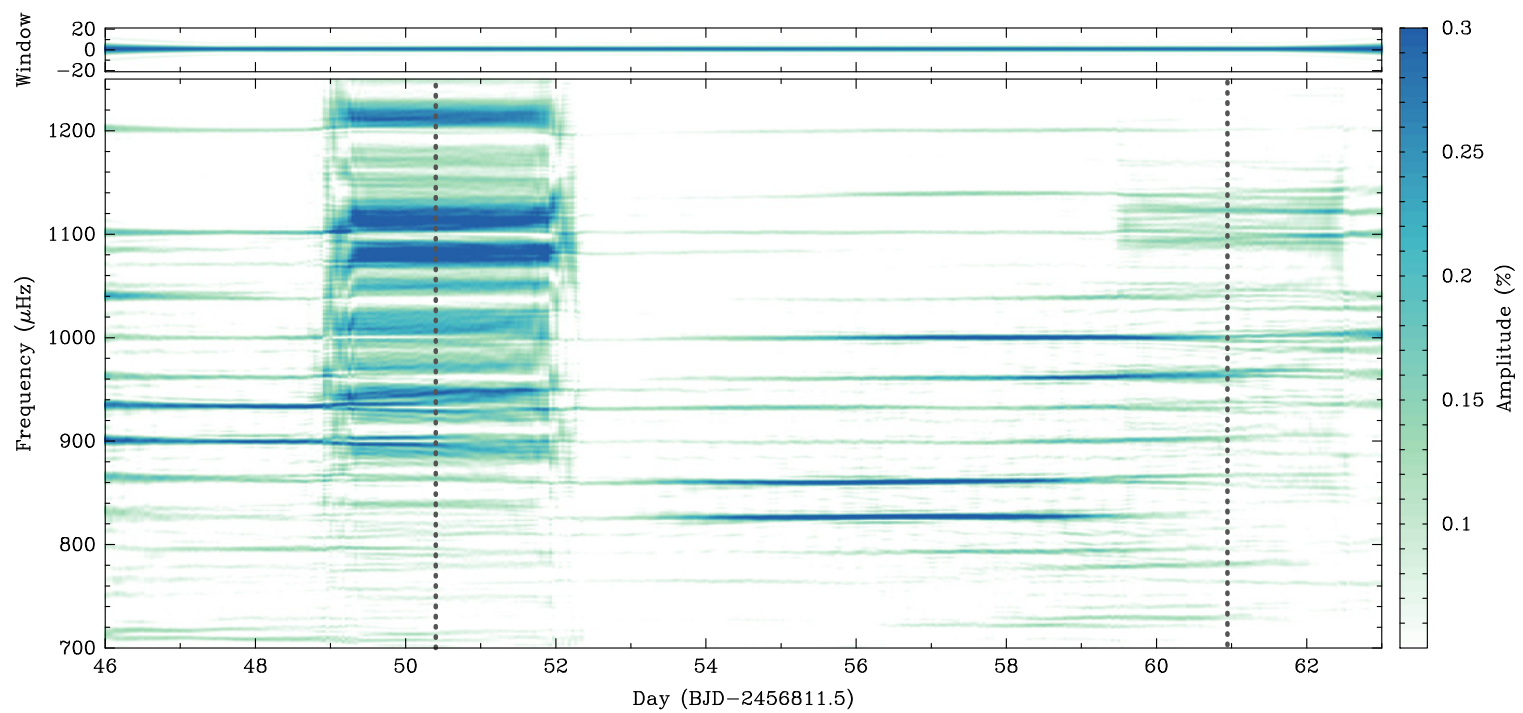

Figure 5. Running FT of the $K 2$ light curve near the seventh outburst shown in Figure 4. Vertical dotted lines mark the maximum brightness during outburst; our 3 -day sliding window smears the events. This plot tracks the post-outburst frequencies, many of which have growing amplitudes until the onset of the eighth outburst peaking near Day 61.

the duration and recurrence of the outbursts are clearly much longer than this. This likely rules out magnetic reconnection events as responsible for the outbursts, since the rise times of magnetic flares occur at roughly the dynamical timescale, and the rise times of the outbursts here are of order hours.

On the other hand, thermal processes can occur on longer timescales. To explore this more quantitatively, we have computed a theoretical white dwarf envelope appropriate to this star $(11,000 \mathrm{~K}, \log g=8.0$, ML2 $/ \alpha=1.0)$ using the WarsawNew Jersey stellar envelope code (Pamyatnykh 1999). We find that the thermal timescale corresponding to the average duration of the outbursts, $14.5 \mathrm{hr}$, occurs at a depth of $10^{-11.5}$ of the star by mass, placing it slightly deeper but near the base of the convection zone.

We note that the recurrence timescale of roughly 8 days is mapped to a deeper layer, the outer $10^{-10}$ of the star by mass. It is possible that processes at these depths play a role in the outbursts. However, the recurrence timescale probably rules out runaway thermonuclear events as the source of the temporary brightness increases, since the equilibrium temperature in this region is below $10^{6} \mathrm{~K}$, significantly too low for nuclear burning.

An occasional deposition of asteroids onto the surface of the white dwarf could contribute the energy required to match the brightness increases, and we now have compelling evidence that disrupted planetesimals frequently pollute the atmospheres of white dwarfs (e.g., Koester et al. 2014). However, this pollution causes metal absorption, which we can rule out from spectroscopy (Voss et al. 2006).

Since the only two known outbursting white dwarfs have very similar effective temperatures and pulsation spectra, it is logical to suspect a connection between the events and the pulsations and the deep convection zone that drives them, especially since we have demonstrated here that the pulsations change before, during, and after outbursts.

We consider a model where the outbursts represent a temporary, rapid reassignment of kinetic energy away from pulsations. It is possible to transfer energy between pulsation modes through resonant mode coupling (Dziembowski 1982), which has previously been invoked to explain the amplitude evolution of $\delta$ Scuti stars in the Kepler mission (Breger \& Montgomery 2014). We note that there is still considerable kinetic energy in the modes which are damped in radiative regions of the star as well as from turbulent dissipation in the convection zone.

A theoretical framework exists for parametric instability via mode coupling of white dwarf pulsations, in which energy is rapidly channelled from an observed, overstable parent mode to damped daughter modes (Wu \& Goldreich 2001). It is possible that the energy of a mode (or multiple modes) grows linearly until it reaches a critical threshold, after which it enters a nonlinear regime and rapidly transfers its energy to resonant daughter modes, or a cascade of resonant daughter modes. These daughter modes may be quickly damped by turbulence in the convection zone and thus deposit their newfound energy there. However, more detailed energetics calculations are required to determine if there is sufficient energy in one (or even multiple) modes to power the observed luminosity increases during outburst.

\subsection{Conclusions}

We present confirmation of a new phenomenon in pulsating white dwarfs by analyzing the second case of outbursts in a cool DAV observed by the Kepler spacecraft, PG $1149+057$. We suggest that these outbursts are connected with the pulsations, perhaps as a result of nonlinear mode coupling.

Still, exciting questions remain. Why have we not previously seen outbursts in extensively studied cool DAVs, such as GD 154 and G29-38 (Pfeiffer et al. 1996; Kleinman et al. 1998)? How do the horizontal surface velocities change from quiescence to outburst (e.g., Van Kerkwijk et al. 2000)? If resonant mode coupling of pulsations is responsible, what mechanism triggers the outburst? Are the outbursts responsible for the eventual cessation of pulsations and the empirical red edge of the DAV instability strip?

Theory can reasonably reproduce the onset of pulsations observed at the blue edge of the DAV instability strip (Brickhill 1991b; Wu \& Goldreich 1999). However, there is still not a clear picture for the red edge where pulsations shut 
down, which is observed empirically to occur at roughly $10,500 \mathrm{~K}$ for a canonical $0.6 M_{\odot}$ white dwarf (Gianninas et al. 2014). Current non-adiabatic calculations predict a much cooler red edge, below $6000 \mathrm{~K}$ (Van Grootel et al. 2012).

Hansen et al. (1985) proposed that longer-period modes would not be reflected off the surface, setting a condition for the red edge. Subsequently, Brickhill (1991b) and Goldreich \& Wu (1999b) suggested that turbulent viscosity in the convection zone may damp the longest-period modes and thus define the red edge. However, neither theory provides a complete picture. Perhaps this newly discovered outburst phenomena plays some role in shutting down pulsations in cool DAVs.

Long-timescale monitoring is necessary to catch and constrain future outbursts from cool pulsating white dwarfs, and it is a testament to the utility of the Kepler instrument to non-planetary science that these events were first discovered thanks to its unblinking gaze. Fortunately, the extended Kepler mission continues its tour of new $K 2$ fields along the ecliptic every three months, offering the possibility to survey additional cool DAVs in the coming years. We look forward to future theoretical and observational constraints to this unexpected phenomenon.

We acknowledge useful discussions with Tom Marsh, Jim Fuller, and Yanqin $\mathrm{Wu}$, and a thoughtful report from an anonymous referee. We thank Simon Walker for sharing the WASP light curve of PG 1149+057 and thank Detlev Koester for making his DA atmospheric models freely available. J.J.H., P.C., and B.T.G. acknowledge funding from the European Research Council under the European Union's Seventh Framework Programme (FP/2007-2013) / ERC Grant Agreement n. 320964 (WDTracer). K.J.B., M.H.M., and D.E.W. gratefully acknowledge the support of the NSF under grant AST-1312983. M.H.M. acknowledges the support of NASA under grant NNX12AC96G. Funding for the Kepler mission is provided by the NASA Science Mission Directorate.

\section{REFERENCES}

Althaus, L. G., Córsico, A. H., Isern, J., \& García-Berro, E. 2010, A\&ARv, 18,471

Armstrong, D. J., Kirk, J., Lam, K. W. F., et al. 2015, A\&A, 579, A19

Barber, S. D., Patterson, A. J., Kilic, M., et al. 2012, ApJ, 760, 26

Barentsen, G. 2015, Astrophysics Source Code Library, record ascl:1503.001

Bell, K. J., Hermes, J. J., Bischoff-Kim, A., et al. 2015, ApJ, 809, 14

Breger, M., \& Montgomery, M. H. 2014, ApJ, 783, 89

Brickhill, A. J. 1991a, MNRAS, 251, 673

Brickhill, A. J. 1991b, MNRAS, 252, 334

Cushing, M. C., Rayner, J. T., \& Vacca, W. D. 2005, ApJ, 623, 1115

Dziembowski, W. 1982, AcA, 32, 147

Fontaine, G., \& Brassard, P. 2008, PASP, 120, 1043

Gianninas, A., Bergeron, P., \& Ruiz, M. T. 2011, ApJ, 743, 138

Gianninas, A., Dufour, P., Kilic, M., et al. 2014, ApJ, 794, 35

Girven, J., Gänsicke, B. T., Steeghs, D., \& Koester, D. 2011, MNRAS, 417, 1210

Goldreich, P., \& Wu, Y. 1999a, ApJ, 511, 904

Goldreich, P., \& Wu, Y. 1999b, ApJ, 523, 805

Greiss, S., Gänsicke, B. T., Hermes, J. J., et al. 2014, MNRAS, 438, 3086

Hansen, C. J., Winget, D. E., \& Kawaler, S. D. 1985, ApJ, 297, 544

Hermes, J. J., Charpinet, S., Barclay, T., et al. 2014, ApJ, 789, 85

Hermes, J. J., Gänsicke, B. T., Bischoff-Kim, A., et al. 2015, MNRAS, 451, 1701

Hermes, J. J., Mullally, F., Østensen, R. H., et al. 2011, ApJL, 741, L16

Howell, S. B., Sobeck, C., Haas, M., et al. 2014, PASP, 126, 398

Kanaan, A., Kepler, S. O., \& Winget, D. E. 2002, A\&A, 389, 896

Kleinman, S. J., Nather, R. E., Winget, D. E., et al. 1998, ApJ, 495, 424

Koester, D. 2010, MmSAI, 81, 921

Koester, D., Gänsicke, B. T., \& Farihi, J. 2014, A\&A, 566, A34

Maxted, P. F. L., \& Marsh, T. R. 1999, MNRAS, 307, 122

Montgomery, M. H., Provencal, J. L., Kanaan, A., et al. 2010, ApJ, 716, 84

Morrissey, P., Conrow, T., Barlow, T. A., et al. 2007, ApJS, 173, 682

Mukadam, A. S., Montgomery, M. H., Winget, D. E., Kepler, S. O., \& Clemens, J. C. 2006, ApJ, 640, 956

Pamyatnykh, A. A. 1999, AcA, 49, 119

Pesnell, W. D. 1985, ApJ, 292, 238

Pfeiffer, B., Vauclair, G., Dolez, N., et al. 1996, A\&A, 314, 182

Renedo, I., Althaus, L. G., Miller Bertolami, M. M., et al. 2010, ApJ, 717, 183

Tremblay, P.-E., Ludwig, H.-G., Steffen, M., \& Freytag, B. 2013, A\&A, 559, AA104

Van Grootel, V., Dupret, M.-A., Fontaine, G., et al. 2012, A\&A, 539, A87

Van Kerkwijk, M. H., Clemens, J. C., \& Wu, Y. 2000, MNRAS, 314, 209

Voss, B., Koester, D., Østensen, R., et al. 2006, A\&A, 450, 1061

Winget, D. E., \& Kepler, S. O. 2008, ARA\&A, 46, 157

Wu, Y., \& Goldreich, P. 1999, ApJ, 519, 783

Wu, Y., \& Goldreich, P. 2001, ApJ, 546, 469 